INPLASY

PROTOCOL

To cite: Yu et al. Association Between Obstructive Sleep Apnoea And T2DM: A DoseResponse Meta-Analysis. Inplasy protocol 2020110027. doi:

10.37766/inplasy2020.11.0027

Received: 07 November 2020

Published: 08 November 2020

Corresponding author:

Zhixiang Yu

xuebojun51@163.com

Author Affiliation:

The Air Force Military Medical University

Support: 2017YFC1309803, 2017 YFC1309804.

Review Stage at time of this submission: Data analysis.

Conflicts of interest:

No conflict of interest.

\section{Association Between Obstructive Sleep Apnoea And T2DM: A Dose- Response Meta-Analysis}

\author{
Yu, Z1; Ji, Q2; Yi, F3; Cheng, J4.
}

Review question / Objective: P: the middle-aged people;: the people with OSA;C: the people without OSA;O: the people got T2DM; S: meta-analysis.

Condition being studied: The refractory hypoxemia episodes character aims/introduction Obstructive sleep apnoea (OSA) in the sleep time. The patients with OSA suffered from comprehensive oxidative stress in all systems. T2DM is a result of metabolism disorder which might be induced by OSA. In this passage, we are exploring the dose-response relationship between OSA and T2DM.

INPLASY registration number: This protocol was registered with the International Platform of Registered Systematic Review and Meta-Analysis Protocols (INPLASY) on 08 November 2020 and was last updated on 08 November 2020 (registration number INPLASY2020110027).

\section{INTRODUCTION}

Review question / Objective: P: the middleaged people;: the people with OSA;C: the people without OSA;O: the people got T2DM; S: meta-analysis.

Condition being studied: The refractory hypoxemia episodes character aims/ introduction Obstructive sleep apnoea (OSA) in the sleep time. The patients with OSA suffered from comprehensive oxidative stress in all systems. T2DM is a result of metabolism disorder which might be induced by OSA. In this passage, we are exploring the dose-response relationship between OSA and T2DM. 


\section{METHODS}

Participant or population: the T2DM patients with OSA and without OSA.

Intervention: The people with OSA.

Comparator: T2DM patients with OSA and without OSA.

Study designs to be included: Observational study.

Eligibility criteria: In the present study, we included cross-sectional studies, cohort studies and case-control studies that had clear outstanding and reported odds ratio (OR) or relative risk (RR) and $95 \%$ confidence interval $(\mathrm{Cl})$ for the association between OSA and T2DM. The researchers used $\mathrm{AHI}$ as the indicators were included. The studies included were all designed to be divided into subgroups by the $\mathrm{AHI}$ and the mean $\mathrm{AHI}$ dose of each subgroup were clear declared. There were no restrictions on gender and age. We excluded patients with type 1 T2DM, and researchers used other indexes as indicators. As for the research type, case reports and reviews were also excluded.

Information sources: PubMed, Embase, Cochran Library, and CNKI (Chinese) databases.

Main outcome(s): Six observational studies were included in the researcher. We excluded a study in the conventional metaanalysis. In the subgroup analysis, mild dose AHI increased the risk of T2DM (odds ratio $=1.32,95 \%$ confidence interval 1.03-1.68, $\mathrm{P}<0.001$ ). Moderate dose AHI increased the risk of T2DM with a higher odds ratio (OR=2.25, 95\% Cl 1.91-2.66, $P<0.001)$. Furthermore, the results of the spline and linear dose-response metaanalysis revealed that the risk of T2DM increased with increasing AHI value.

Quality assessment / Risk of bias analysis: We evaluated the included studies according to the Newcastle-Ottawa scale (NOS). A quantitative scoring device proposed by the Cochrane Collaboration was adopted for assessing the methodological quality of the studies. The NOS contains three significant spaces: selecting subjects, comparability between bunches, and outcome measures. The most extreme of each region is four, two, and three. The lower the full score of the three parts, the worse the article is in methodological quality.

Strategy of data synthesis: Two investigators (YZX, YF) finished the conventional meta-analyses with Cochrane review manager version 5.3 software's help to assess a specific outcome's risk.

Subgroup analysis: The subgroup was divided into mild-dose(5<AHI15).

Sensibility analysis: The evaluation of heterogeneity among studies was undergone with the use of $Q$ and 12 . We adopt a standard for $P$-value that $P$ value $<0.1$ means the results possessed statistical heterogeneity. 12 describes the extent of variation due to heterogeneity rather than chance. The lower 12 is, the less variation is. $12<25 \%$ was considered little heterogeneity, $25 \%<1250 \%$ showed there existed enough heterogeneity to select a random effects-model. While $12<50 \%$, a fixed-effect model was employed.

Country(ies) involved: China.

Keywords: T2DM, OSA, Dose-Response Meta-Analysis.

Contributions of each author:

Author 1 - Zhixiang Yu - The author performed the analysis and wrote the manuscript together.

Email: xuebojun51@163.com

Author 2 - Qiuhe Ji - The author conceived the ideas.

Email: qiuheji@hotmail.com

Author 3 - Fu Yi - The author gave guidance for the analysis.

Email: yi12fu56@hotmail.com

Author 4 - Jinxiang Cheng - The author performed the analysis and wrote the manuscript together.

Email: chengjinxiang423@foxmail.com 